\title{
Protective effects of PACAP against lactacystin-induced PC12 cell apoptosis due to prevention of mitochondrial damage and endoplasmic reticulum stress.
}

\author{
Lina Guan" ${ }^{1 \#, ~ Y o n g q i a n g ~} \mathrm{Ji}^{2 \#}$, Guoping $\mathrm{Yu}^{3 *}$, Chao Ren ${ }^{3}$, Jie Liu', Maowen $\mathrm{Ba}^{3}$, Peiwen Lian ${ }^{4}$, Ning $\mathrm{Li}^{4}$ \\ ${ }^{1}$ Department of Neurology, Intensive Care Unit, the Affiliated Yantai Yuhuangding Hospital of Qingdao University, \\ Yantai, PR China \\ ${ }^{2}$ Department of Nephrology, the Affiliated Yantai Yuhuangding Hospital of Qingdao University, Yantai, PR China \\ ${ }^{3}$ Department of Neurology, the Affiliated Yantai Yuhuangding Hospital of Qingdao University, Yantai, PR China \\ ${ }^{4}$ Central Laboratory, the Affiliated Yantai Yuhuangding Hospital of Qingdao University, Yantai, PR China \\ \#These authors contributed equally to this work
}

\begin{abstract}
Background: Pituitary Adenylate Cyclase Activating Peptide (PACAP) has a significant protective effect on nerve cell damage induced by several neurotoxins. The ubiquitin proteasome inhibitor lactacystin, one new neurotoxin, could cause cell damage by inducing dopaminergic cells apoptosis. Whether PACAP has a protective effect on lactacystin-induced cytotoxic model has not been reported.

Aims: The present study was designed to explore whether PACAP can effectively inhibit the lactacystininduced dopaminergic apoptosis.

Methods: Pheochromocytes (PC12 cells) differentiated by nerve growth factor as neurons were treated with lactacystin $(20 \mu \mathrm{mol} / \mathrm{l})$ for $24 \mathrm{~h}$, to establish the Parkinson's disease cells model. Groups are set up as control group, lactacystin group, PACAP1-27 intervention group, PACAP1-27 and PACAP6-27 cointervention group.

Results: Treatment with lactacystin can make the cell vitality obviously decline. Compared with control group, cell morphology presented obviously damaged change in lactacystin group. After treatment with lactacystin, the expression of mitochondrial bcl-2 dramaticly decreased, expression of mitochondrial bax had no change, the rate of bcl-2/bax dramaticly decreased, and thus expression of caspase-3 increased. Meanwhile, caspase-12, the characteristic marker of endoplasmic reticulum stress, increased significantly in lactacystin group. However, these changes could be partly reversed by treatment with PACAP1-27. This protective effect of PACAP1-27 was negated by PACAP6-27, a receptor antagonist of PACAP1-27.

Conclusion: Lactacystin led to cell damage by inducing mitochondrial damage and endoplasmic reticulum stress; PACAP1-27 played a protective role by regulating the mitochondrial and endoplasmic reticulum related dual signal pathway. As one PACAP1-27 receptor antagonist, PACAP6-27 attenuated this effect of PACAP1-27.
\end{abstract}

Keywords: Lactacystin, PACAP, Bcl-2, Bax, Caspase-3, Caspase-12. Abbreviations: PACAP: Pituitary Adenylate Cyclase Activating Peptide; PC12 Cells: Pheochromocytes.

Accepted on November 14, 2017

\section{Introduction}

Parkinson's disease is a clinic common neurodegenerative disorder, and characterized by the progressive loss of dopaminergic neurons in the substantia nigra. There is still no effective therapies [1]. In order to investigate the pathogenesis of Parkinson's disease and find effective treatment, we need to establish experimental Parkinson's disease cell model. Many scholars used methyl-phenyl-tetrahydropyridine and 6- hydroxy-dopamine for preparation of Parkinson's disease cell model, but both models are characterized with lack of Lewy body. Rideout et al. [2] applied lactacystin in bare PC12 cells and PC12 cells differentiated by nerve growth factor, and found a dose-related drug-induced cell death, the death can be inhibited by caspase inhibitor. They also found that the ubiquitin-stained positive inclusion bodies in living cells, and human $\alpha$-synuclein homologous synuclein-1 staining also 
showed positive results. The cell model induced by lactacystin mimics the Lewy bodies of the Parkinson's disease and apoptosis [3-5].

PACAP is a neuropeptide with multiple effects, and belongs to the secretin/glucagon/vasoactive intestinal peptide family. There are two structural forms: PACAP27 and PACAP38. PACAP27 can spread freely across the blood-brain barrier in non-saturation manner [6]. PACAP27 may have potential practical value. PACAP27 has two forms: PACAP1-27 and PACAP6-27. Some studies have found that PACAP1-27 has a significant protective effect on nerve cell damage induced by excitatory amino acids, $\beta$-amyloid and prion protein [7]. But whether PACAP1-27 has a protective effect in lactacystininduced cytotoxic model has not been reported. Therefore, the study explored the protective effect of PACAP1-27 and its related molecular mechanisms in lactacystin-induced cytotoxic model.

\section{Materials and Methods}

This was designed as a controlled cell study. Experiments were performed at the central laboratory, Yantai Yuhuangding Hospital, Yantai, PR China, between April 2011 and November 2013.

\section{Materials}

10\% fetal bovine serum (Australia NQBB Company); 1640 culture medium (China HyClone Company); Nerve growth factor (China HyClone Company); Lactacystin (British Cayman Company); Rabbit anti-rat caspase-3 antibody (English abcam Company); Rabbit anti-rat caspase-12 antibody (English abcam Company); Rabbit anti-rat bcl-2 antibody (Cell Signaling company American); Rabbit anti-rat bax antibody (U.S. Cell Signaling Company); GAPDH antibody (English abcam Company); PACAP1-27 (U.S. Tocris Bioscience Corporation American); PACAP6-27 (U.S. Biological Corporation American); MTT (Sigma USA); Bicinchoninic acid protein assay kit (U.S. Pierce Company).

\section{Methods}

Cell culture: PC12 cells supplemented with $10 \%$ fetal bovine serum and 1640 culture medium were cultured at 5\% carbon dioxide, $37^{\circ} \mathrm{C}$ incubator. The medium was changed every other day. PC12 cell was induced by $7 \mathrm{~d}$ treatment with nerve growth factor $(0.1 \mathrm{ng} / \mathrm{l})$ to neuron-like cells.

Drug treatment: The cells were divided into four groups: control group, lactacystin group, PACAP1-27 intervention group, PACAP1-27 and PACAP6-27 co-intervention group.

PC12 cells differentiated by nerve growth factor $(0.1 \mathrm{ng} / \mathrm{l})$ for $7 \mathrm{~d}$ as the control group, then added to lactacystin of different concentrations $(5,10,20,40 \mu \mathrm{mol} / \mathrm{l})$ processing PC12 cells separately for $0,6,12,24 \mathrm{~h}$, the cell viability present concentration and time dependent-on decline, lactacystin of 20 $\mu \mathrm{mol} / 1$ for $24 \mathrm{~h}$ can make the cell vitality decline about $50 \%$.
Then the $20 \mu \mathrm{mol} / 1$ lactacystin for $24 \mathrm{~h}$ was selected for the lactacystin group in the study.

Control group: PC12 cells differentiated by nerve growth factor $(0.1 \mathrm{ng} / \mathrm{l})$ for $7 \mathrm{~d}$.

Lactacystin group: PC12 cells differentiated by nerve growth factor $(0.1 \mathrm{ng} / \mathrm{l})$ for $7 \mathrm{~d}$, then added to $20 \mu \mathrm{mol} / 1$ lactacystin for $24 \mathrm{~h}$.

PACAP1-27 intervention group: $\mathrm{PC} 12$ cells of lactacystin group added to PACAP1-27 $\left(10^{-8}\right)$ for $24 \mathrm{~h}$. PACAP1-27 and PACAP6-27 co-intervention group: PC12 cells of lactacystin group added to PACAP1-27 $\left(10^{-8}\right)$ and PACAP6-27 $\left(10^{-5}\right)$ for $24 \mathrm{~h}$.

MTT assay: The viability of cultured cells was evaluated using the MTT colorimetric assay. MTT is an indicator of mitochondrial respiration and MTT reduction is mediated by active mitochondrial succinate dehydrogenase in living cells, which has commonly been used to infer cellular viability. MTT reduction was quantified at $570 \mathrm{~nm}$ using a micro-plate reader.

Cell morphology: After exposed to drugs for $24 \mathrm{~h}$, each group was placed under inverted microscope to observe cell morphology, to observe changes of neurons and axons.

Western blots: Cells were collected and washed with ice-cold PBS and lysed in lysis buffer. After incubation for $20 \mathrm{~min}$ on ice, cell lysates were centrifuged $\left(10,000 \mathrm{~g}\right.$ for $10 \mathrm{~min}$ at $\left.4^{\circ} \mathrm{C}\right)$ and the protein concentration in the extracts was determined using a bicinchoninic acid protein assay kit. Samples were resuspended in Sodium Dodecyl Sulfate (SDS)-polyacrylamide gel electrophoresis sample buffer and boiled for $5 \mathrm{~min}$. A volume of $20 \mu \mathrm{l}$ of solubilized total cell lysate (15 $\mu \mathrm{g}$ protein) was loaded per lane of a $10 \%$ polyacrylamide gel for carrying out SDS-polyacrylamide gel electrophoresis, followed by transfer onto a polyvinylidine fluoride membrane at $120 \mathrm{~V}$ for $120 \mathrm{~min}$ at $4^{\circ} \mathrm{C}$ using a Mini Trans-Blot Cell apparatus. Membranes were blocked for $60 \mathrm{~min}$ at room temperature $\left(25^{\circ} \mathrm{C}\right)$ using $5 \%$ skimmed milk powder in Tris-buffered saline (TBS; $10 \mathrm{mM}$ Tris- $\mathrm{HCl}, 150 \mathrm{mM} \mathrm{NaCl}, \mathrm{PH} 7.4$ ) and then probed with GAPDH antibody, rabbit anti-rat caspase-3 (1: 500), caspase-12 antibody (1: 500) and bcl-2 (1:500), bax antibody (1: 500) overnight at $4{ }^{\circ} \mathrm{C}$ The blots were washed three times in TBS-T (with $0.05 \%$ tween-20) at room temperature and then incubated with horseradish peroxidaseconjugated secondary antibody diluted in TBS-T $(1: 2,000)$ for $1 \mathrm{~h}$ at room temperature, followed by washing four times. Signals were detected with a chemiluminescence kit. The blots were then removed from the working solution and exposed to Kodak Biomax MS-1 films (Sigma) for 0.5-3 min. Quantitative analysis of Western blots was carried out by calculating the relative density of the target bands. To reduce the differences, values of each band of sample were compared with that of GAPDH.

\section{Statistics}

Statistical analysis was carried out using SAS 6.12 statistical software, Data was expressed as mean $\pm \mathrm{SD}$, Groups were 
compared using analysis of variance, pairwise comparisons using LSD-t-test, $\quad \mathrm{P}<0.05$ was considered statistically significant.

\section{Results}

\section{The cell viability of each group}

After exposed to drugs for $24 \mathrm{~h}$, the cell viability of each group was measured. Compared with control group (100\%), cell activity of lactacystin group (50.1\%) decreased, the cell viability of PACAP1-27 intervention group (77.8\%) was enhanced compared with lactacystin group, and there was little difference between PACAP1-27 and PACAP6-27 cointervention group $(55.6 \%)$ and lactacystin group, the cell viability of PACAP1-27 and PACAP6-27 co-intervention group was lower than PACAP1-27 intervention group, the difference was statistically significant $(\mathrm{P}<0.01)$ (Figure 1).

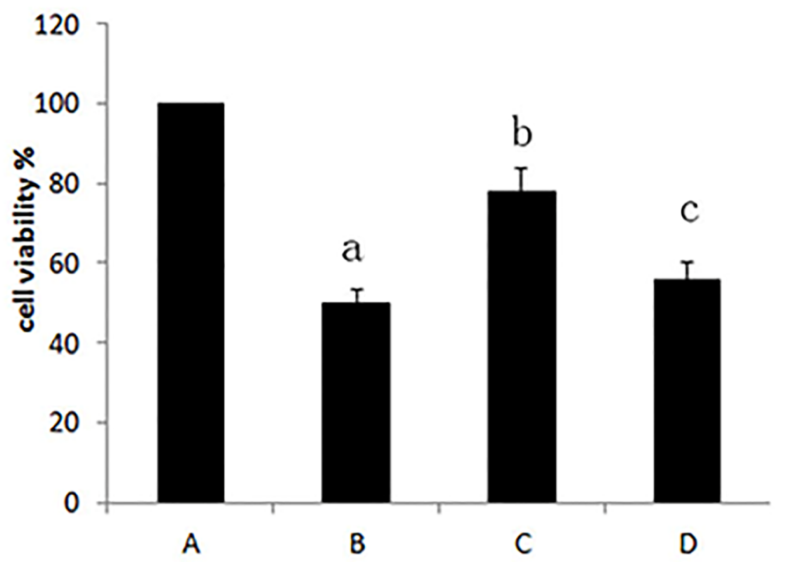

Figure 1. Cell viability changes of each group. A: Control group B: Lactacystin group C: PACAP1-27 intervention group D: PACAP1-27 and PACAP6-27 co-intervention group. ${ }^{a} P<0.01$, vs. control group; ${ }^{b} P<0.01$, vs. lactacystin group; ${ }^{c} P<0.01$, vs. $P A C A P 1-27$ intervention group.

\section{PC12 cell morphology}

Pretreated with $20 \mu \mathrm{mol} / \mathrm{l}$ lactacystin for $24 \mathrm{~h}$, PC12 cell morphology changed significantly (Figure 2). The cells of control group showed active proliferative capacity and had larger size with significantly longer neurites. In lactacystin group, cell size became smaller and neurites became shorter or disappeared, and cell proliferative capacity declined. Compared with lactacystin group, cell damage change in PACAP1-27 intervention group was improved more obviously, and the number of survival cells increased. In PACAP1-27 and PACAP6-27 co-intervention group, the growth state of the cells was not as good as that of PACAP1-27 intervention group, but there was no difference with lactacystin group.

\section{Western blot analysis of apoptosis protein expression of each group}

Compared with control group, the expression of bcl-2 in lactacystin group dramatically decreased $(\mathrm{P}<0.01)$, bax expression had no change, the ratio of bcl-2/bax dramatically decreased $(\mathrm{P}<0.01)$, caspase- 3 and caspase-12 activity increased significantly $(\mathrm{P}<0.01)$. Compared with lactacystin group, expression of bcl-2 in PACAP1-27 intervention group dramatically increased $(\mathrm{P}<0.01)$, bax expression had no change, the ratio of bcl-2/bax dramatically increased $(\mathrm{P}<0.01)$, meanwhile the activity of caspase- 3 and caspase- 12 was significantly downregulated $(\mathrm{P}<0.01)$; the effect of PACAP1-27 was negated by PACAP6-27 (Figure 3).
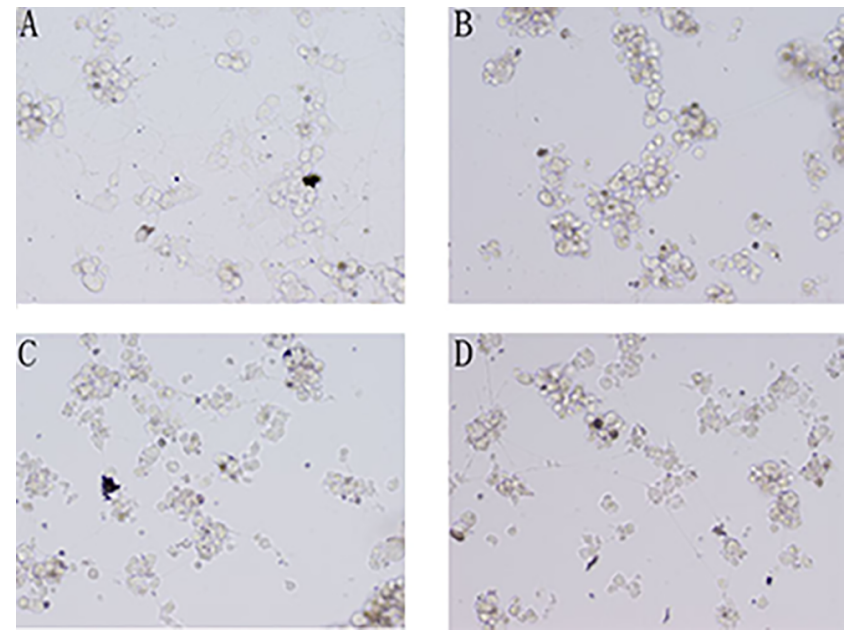

Figure 2. Cell morphology of each group. A: Control group; B: Lactacystin group; C: PACAP1-27 intervention group; D: PACAP1-27 and PACAP6-27 co-intervention group. With drugs treating for $24 \mathrm{~h}, \mathrm{PC} 12$ cell morphology changes of each group (Olympus inverted microscope X200).

\section{Discussion}

Parkinson's disease models with Lewy bodies established using lactacystin reveals the proteasome function of substantia nigra cells impaired, not only cannot clear $\alpha$-synuclein and other proteins of cytoplasm in time, but also is an important factor in the formation of Lewy bodies and substantia nigra degeneration eventually led to Parkinson's disease, and provides an ideal experimental model for further research [2].

The study found that the impact of lactacystin on the vitality of PC12 cells showed a dose-dependent-on decline, and that toxic effects occur at low dose $(5 \mu \mathrm{mol} / 1)$, and verify that low-dose exposure to lactacystin can cause cell damage; the cell death induced by lactacystin is mainly karyopyknosis apoptosis observing by inverted microscope, further confirmed the neurotoxic effects of lactacystin can cause apoptosis, and lactacystin is an ideal Parkinson's disease-inducing agent in vitro cell model. 


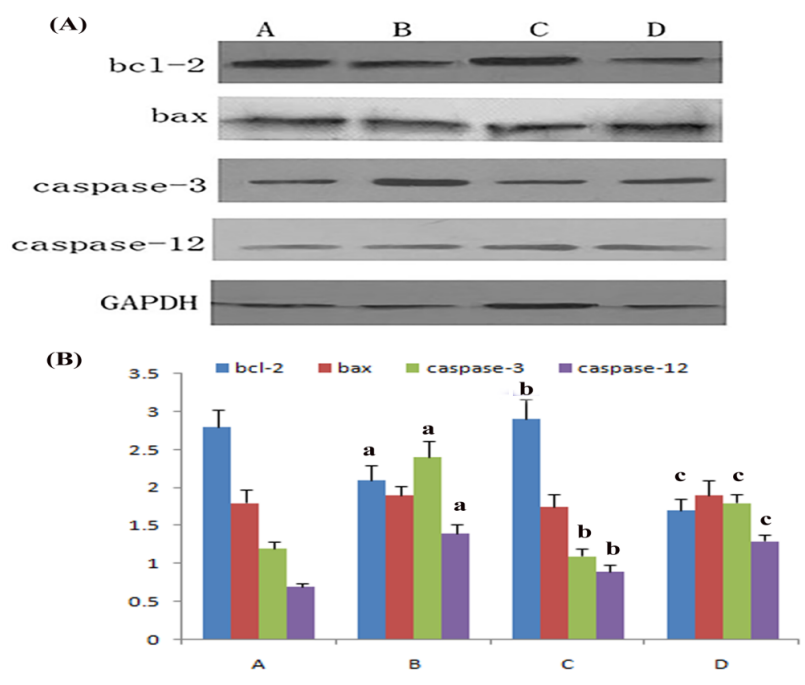

Figure 3. Analysis of apoptosis protein expression in each group. (A)Western blot analysis of bcl-2, bax, caspase-3 and caspase-12 is compared between groups. (B) Bands corresponding to bcl-2, bax, caspase-3 and caspase-12 were scanned and their optical density quantified by densitometry. A: Control group; B: Lactacystin group; C: PACAP1-27 intervention group; D: PACAP1-27 and PACAP6-27 co-intervention group. ${ }^{a} P<0.01$, vs. control group; ${ }^{b} P<0.01$, vs. lactacystin group; ${ }^{c} P<0.01$, vs. $P A C A P 1-27$ intervention group.

PACAP is an endogenous neuropeptide with a diverse array of biological functions including anti-apoptosis, antiinflammation and anti-oxidative stress [8-10]. And PACAP plays the anti-apoptosis role by regulating the cell expression of apoptosis-related protein caspase-3, bcl-2, bax, caspase-12 etc. [11]. PACAP is widely distributed in the central and peripheral nervous system [9]. PACAP is non-toxic in its own, and can easily cross the blood-brain barrier from the circulation into the brain parenchyma in a very short time following intravenous or intraventricular injection. Therefore, PACAP is a powerful emerging candidate as a therapeutic for neurodegenerative diseases. Different neurotoxins can cause caspase-3 activity increase and finally damage to cells [12], PACAP1-27 can inhibit this increase of response $[13,14]$. Our results show that lactacystin significantly increase the caspase-3 activity in PC12 cells, PACAP1-27 can indeed inhibit this increase. We found that this protective effect can be reversed by specific antagonist PACAP6-27 of PACAP/VIP typeI receptor (i.e. PAC1), and previous studies have found PAC1 receptor mRNA expression in PC12 cells does exist $[13,15,16]$, we speculate that the inhibition effect of PACAP27 on the cell apoptosis induced by lactacystin may be mainly mediated by PAC1 receptor, and PAC1 receptors are the key parts of lactacystin-induced apoptosis process. Meanwhile, the study further discussed the involved molecular mechanisms of the protective effect of PACAP1-27. It is known that mitochondria-mediated pathway is accounted for the most important position in apoptosis [17-19].

There are soluble proteins in the mitochondria, such as cytochrome C. Once triggered, a large number of neurotoxic factors can cause increased permeability of the mitochondrial outer membrane [20], bcl-2 protein family are important effectors of the mitochondrial outer membrane permeability [21], capable of forming lipid pore enough for cytochrome $\mathrm{C}$ to transverse and release into the cytoplasm, resulting in caspase-3 activation and apoptosis [22,23]. Intracellular ratio of bcl-2 and bax regulates the happen of apoptosis. This study confirmed that expression of bcl-2 decreased after treatment of lactacystin, bax expression levels did not change, bcl-2/bax ratio decreased. We can speculate lactacystin make mitochondrial permeability transition pore opening and mitochondria releasing cytochrome $\mathrm{C}$ by inhibiting the expression of bcl-2, reducing bcl-2/bax ratio, inducing bax activation and shifting to the mitochondria, which may further trigger caspases cascade reaction leading to apoptosis. PACAP could reverse the toxic effect of lactacystin, as demonstrated in the results. These findings indicated that PACAP played a protective role by regulating the mitochondrial related signal pathway.

Except for the classic death receptor pathway and mitochondrial pathway, the endoplasmic reticulum apoptosis signaling pathway has also attracted wide attention [24,25]. Caspase-12 is a specific marker of activated endoplasmic reticulum stress inducing apoptosis [26]. Caspase-12 is present in cytoplasmic side of the endoplasmic reticulum membrane with the form of zymogen, and is specifically activated in a state of endoplasmic reticulum stress. Once caspase-12 is activated, it acts on downstream effector caspases, inducing apoptosis [27,28]. This study shows the expression of activating caspase-12 protein significantly increased $24 \mathrm{~h}$ after lactacystin treatment, and was paralleled with the change of apoptosis rate, suggesting that lactacystin also can induce endoplasmic reticulum stress, and then inducing apoptosis. Thus, lactacystin can induce apoptosis in experimental Parkinson's disease cell model through induction of mitochondrial damage by the activation of caspase-3, bcl-2 etc., and also can induce apoptosis through the induction of endoplasmic reticulum stress by activation of caspase- 12 .

\section{Conclusion}

Our research for the first time shows that PACAP1-27 can effectively inhibit lactacystin-induced casapase-3 and caspase-12 increases, and PACAP6-27 can antagonize this effect, suggesting that PACAP1-27 plays a protective role by regulating the mitochondrial and endoplasmic reticulum related dual signal pathway. Further studies are required to explore more detailed mechanisms of the observed effects.

\section{Authors' Contributions}

Lina Guan, Guoping Yu, and Yongqiang Ji designed the research, performed the experiments, interpreted the data and wrote the manuscript. Ning Li helped with the interpretation and analysis of the data. Chao Ren supervised, headed and financed the work and helped editing the manuscript. Maowen $\mathrm{Ba}$ and Peiwen Lian supervised the project, designed the research and interpreted the data. All authors read and approved the final manuscript. 


\section{Acknowledgements}

This study was funded by the Shandong Provincial Natural Science Foundation, China (No.ZR2010HM117), Yantai Science and Technology Planning Projects (No.2010148-6) and National Natural Science Foundation of China Projects (No. 81501185), Project 2016WS017 from Yantai Science and Technology Planning Projects. The authors would like to acknowledge the Central laboratory of Yantai Yuhuangding Hospital for the use of their laboratory facilities.

\section{Competing Interests}

The authors declare that they have no competing interests.

\section{References}

1. Athauda D, Foltynie T. The ongoing pursuit of neuroprotective therapies in Parkinson disease. Nat Rev Neurol 2015; 11: 25-40.

2. Zhang KZ, Jiang YP, Ding XS. Applying lactacystin, a selective proteasome inhibitor, to set up the rat model of Parkinsons diasease with Lewy body. Clin Neurol 2006; 19: 358-361.

3. Kerr JF. History of the events leading to the formulation of the apoptosis concept. Toxicology 2002; 27: 471-474.

4. Kerr JF, Wyllie AH, Currie AR. Apoptosis: a basic biological phenomenon with wide-ranging implications in tissue kinetics. Br J Cancer 1972; 26: 239-257.

5. Taylor RC, Cullen SP, Martin SJ. Apoptosis: controlled demolition at the cellular level. Nat Rev Mol Cell Biol 2008; 9: 231-241.

6. Dogrukol-Ak D, Tore F, Tuncel N. Passage of VIP/PACAP/ secretin family across the blood-brain barrier: therapeutic effects. Curr Pharm Des 2004; 10: 1325-1340.

7. Lamine A. Characterizations of a synthetic pituitary adenylate cyclase-activating polypeptide analog displaying potent neuroprotective activity and reduced in vivo cardiovascular side effects in a Parkinsons disease model. Neuropharmacol 2015.

8. Shioda S. Pleiotropic functions of PACAP in the CNS: neuroprotection and neurodevelopment. Ann N Y Acad Sci 2006; 1070: 550-560.

9. Vaudry D. Pituitary adenylate cyclase-activating polypeptide and its receptors: 20 years after the discovery. Pharmacol Rev 2009; 61: 283-357.

10. Reglodi D. Effects of PACAP on in vitro and in vivo neuronal cell death, platelet aggregation, and production of reactiveoxygen radicals. Regul Pept 2004; 123: 51-59.

11. Szabadfi K. PACAP promotes neuron survival in early experimental diabetic retinopathy. Neurochem Int 2014; 64: 84-91.

12. Yuan J, Yankner BA. Apoptosis in the nervous system. Nature 2000; 407: 802-809. Onoue S. The neuropeptide PACAP attenuates beta-amyloid (1-42)-induced toxicity in PC12 cells. Peptides 2002; 23: 1471-1478.
13. Onoue S. PACAP protects neuronal PC12 cells from the cytotoxicity of human prion protein fragment 106-126. FEBS Lett 2002; 522: 65-70.

14. Dichter MA. Nerve growth factor-induced increase in electrical excitability and acetylcholine sensitivity of a rat pheochromocytoma cell line. Nature 1997; 268: 501-504.

15. Lou LR. Pituitary adenylate cyclase-activating polypeptide protects neuro- 2 a cells from $\beta$ amyloid protein cytotoxicity by modulating intracellular calcium. Sheng Li Xue Bao 2003; 55: 42-46.

16. Jamen F. Up-regulation of the PACAP type-1 receptor (PAC1) promoter by neurotrophins in rat PC12 cells and mouse cerebellar granule cells via the Ras/mitogenactivated protein kinase cascade. Neurochem 2002; 82: 1199-1207.

17. Li P. Cytochrome $\mathrm{c}$ and dATP-dependent formation of Apaf-1/caspase-9 complex initiates an apoptotic protease cascade. Cell 1997; 91: 479-489.

18. Liu X. Induction of apoptotic program in cell-free extracts: requirement for dATP and cytochrome c. Cell 1996; 86: 147-157.

19. Zou H. Apaf-1, a human protein homologous to C. elegans CED-4, participates in cytochrome c-dependent activation of caspase-3. Cell 1997; 90: 405-413.

20. Vaux DL. Apoptogenic factors released from mitochondria. Biochim Biophys Acta 2011; 1813: 546-550.

21. Hardwick JM, Soane L. Multiple functions of BCL-2 family proteins. Cold Spring Harb Perspect Biol 2013; 5.

22. Martinou JC, Youle RJ. Mitochondria in apoptosis: Bcl-2 family members and mitochondrial dynamics. Dev Cell 2011; 21: 92-101.

23. Xiong S, Mu T, Wang G, Jiang X. Mitochondria-mediated apoptosis in mammals. Protein Cell 2014; 5: 737-749.

24. Costa RO. ER stress-mediated apoptotic pathway induced by Abeta peptide requires the presence of functional mitochondria. Alzheimers Dis 2010; 20: 625-636.

25. Umeda $\mathrm{T}$. Intraneuronal amyloid $\beta$ oligomers cause cell death via endoplasmic reticulum stress, endosomal/ lysosomal leakage, and mitochondrial dysfunction in vivo. Neurosci Res 2011; 89: 1031-1042.

26. Ishige $\mathrm{K}$. Role of caspase-12 in amyloid beta-peptideinduced toxicity in organotypic hippocampal slices cultured for long periods. Pharmacol Sci 2007; 104: 46-55.

27. Degterev A. A decade of caspases. Oncogene 2003; 22: 8543-8567.

28. Nakagawa T. Caspase-12 mediates endoplasmic-reticulumspecific apoptosis and cytotoxicity by amyloid-beta. Nature 2000; 403: 98-103.

\section{*Correspondence to}

Guoping Yu

Department of Neurology

The Affiliated Yantai Yuhuangding Hospital of Qingdao University

PR China 